\title{
Distribution Inference for Physical and Orbital Properties of Jupiter's Moons
}

\author{
F. B. Gao $\mathbb{D}^{1},{ }^{1}$ X. H. Zhu, ${ }^{2}$ X. Liu, ${ }^{1}$ and R. F. Wang ${ }^{1}$ \\ ${ }^{1}$ School of Mathematical Science, Yangzhou University, Yangzhou 225002, China \\ ${ }^{2}$ Department of Mathematics, Shanghai University, Shanghai 200444, China \\ Correspondence should be addressed to F. B. Gao; gaofabao@sina.com
}

Received 4 June 2018; Revised 1 September 2018; Accepted 30 September 2018; Published 1 November 2018

Academic Editor: Geza Kovacs

Copyright (c) 2018 F. B. Gao et al. This is an open access article distributed under the Creative Commons Attribution License, which permits unrestricted use, distribution, and reproduction in any medium, provided the original work is properly cited.

\begin{abstract}
According to the physical and orbital characteristics in Carme group, Ananke group, and Pasiphae group of Jupiter's moons, the distributions of physical and orbital properties in these three groups are investigated by using one-sample Kolmogorov-Smirnov nonparametric test. Eight key characteristics of the moons are found to mainly obey the Birnbaum-Saunders distribution, logistic distribution, Weibull distribution, and $t$ location-scale distribution. Furthermore, for the moons' physical and orbital properties, the probability density curves of data distributions are generated; the differences of three groups are also demonstrated. Based on the inferred results, one can predict some physical or orbital features of moons with missing data or even new possible moons within a reasonable range. In order to better explain the feasibility of the theory, a specific example is illustrated. Therefore, it is helpful to predict some of the properties of Jupiter's moons that have not yet been discovered with the obtained theoretical distribution inference.
\end{abstract}

\section{Introduction}

There are 69 (the number has been refreshed to 79 by a team from Carnegie Institution for Science in July 2018. https:// sites.google.com/carnegiescience.edu/sheppard/moons/jupitermoons) confirmed moons of Jupiter, around 65 of which have been well investigated $[1,2]$. Considering the formation of Jupiter's moons is influenced by diverse factors, which results in their physical characteristics differing greatly [3], Jupiter's moons are divided into two basic categories: regular and irregular. The regular satellites are so named because they have prograde and near-circular orbits of low inclination, and they are in turn split into two groups: Inner satellites and Galilean [4]. The irregular satellites are actually the objects whose orbits are far more distant and eccentric. They form families that share similar orbits (semi-major axis, inclination, and eccentricity) and composition. These families, which are considered to be part of collisions, arise when the larger parent bodies were shattered by impacts from asteroids captured by Jupiter's gravitational field. That is to say, at the early time of moons' formation of the Jupiter, mass of the original moon's ring was still sufficient to absorb the asteroid's power and put it into orbit. So, part of the irregular moons might be created by the captured asteroids and then collided with other moons $[5,6]$, thus forming the various groups we see today. The identification of satellite families is tentative (please see $[7,8]$ for more details), and these families bear the names of their largest members. The most detailed modelling of the collisional origin of the families was reported in $[9,10]$.

According to this identification scheme [11], 60 moons were classified into 8 different groups, including Small Inner Regulars and Rings, Galileans, Themisto group, Carpo group, Himalia group, Carme group, and Ananke group as well as Pasiphae group, in addition to 9 satellites that do not belong to any of previous groups. The detailed information about all the groups of Jupiter's moons can be found in Appendix A.

In recent years, many scientists have paid considerable attention to astronomical observation, physical research, and deep space exploration of small bodies, including asteroids, comets, and satellites, and so on. For example, planetary scientist Carry collected mass and volume estimates of 17 near-Earth asteroids, 230 main-belt and Trojan asteroids, 12 comets, and 28 trans-Neptunian objects from the known 
literature [12]. The accuracy and biases affecting the methods used to estimate these quantities were discussed and best-estimates were strictly selected. For the asteroids in retrograde orbit, there are at least 50 known moons of Jupiter's that are retrograde, some of which are thought to be asteroids or comets that originally formed near the gas giant and were captured when they got too close. Kankiewicz and Włodarczyk selected the 25 asteroids with the best-determined orbital elements and then estimated their dynamical lifetimes by using the latest observational data, including astrometry and physical properties [13]. However, few researchers have tried to extrapolate the distribution of Jupiter's satellites through statistical methods as yet.

In this paper, distributions of physical and orbital properties for the moons of Jupiter will be conducted by using one-sample Kolmogorov-Smirnov (K-S) test and maximum likelihood estimation [14-16]. Based on the analysis of satellites' data, it is found surprisingly that the physical and orbital characteristics obey some distribution, such as BirnbaumSaunders distribution [17], logistic distribution, Weibull distribution, and $t$ location-scale distribution. Furthermore, the probability density curves of the data distribution are generated, and the differences of physical and orbital characteristics in the three groups are presented. In addition, the results of theoretical inference results are then proved to be feasible through one concrete example. Therefore, the results may be helpful to astronomers to discover new moons of Jupiter in the future.

\section{Method of Distribution Inference}

In statistics, the K-S test, one type of nonparametric test, is used to determine whether a sample comes from a population with a specific distribution. The null hypothesis of onesample K-S test is that the Cumulative Distribution Function (CDF) of the data follows the adopted CDF. For one-sample case, null distribution of statistic can be obtained from the null hypothesis that the sample is extracted from a reference distribution. The two-sided test for "unequal" CDF tests the null hypothesis against the alternative that the CDF of the data is different from the adopted CDF. The test statistic is the maximum absolute difference between the empirical CDF calculated by $x$ and the hypothetical CDF:

$$
D_{n}=\sup _{x}\left|F_{n}(x)-F(x)\right|
$$

where $F(x)$ is a given $\mathrm{CDF}$ and

$$
F_{n}(x)=\frac{1}{n} \sum_{i=1}^{n} I_{(-\infty, x]}\left(X_{i}\right)
$$

is the empirical distribution function of the observations $X_{i}$. Here $I_{(-\infty, x]}\left(X_{i}\right)$ is the indicator function with the following form

$$
I_{(-\infty, x]}\left(X_{i}\right)= \begin{cases}1, & X_{i} \leq x \\ 0, & \text { otherwise. }\end{cases}
$$

According to Glivenko-Cantelli theorem [18], if the sample comes from distribution $F(x)$, then $D_{n}$ will almost surely converge to zero when $n \longrightarrow \infty$. Therefore, we only focus on three satellite groups that have more than ten satellites in their groups, respectively. Of all these groups, Themisto group and Carpo group only contain one satellite, respectively, and only 4 moons were found separately in Small Inner Regulars and Rings, as well as in Galileans group. Moreover, there are 5 moons in Himalia group. For these 5 groups, there is no sufficient data for distribution inference, so we will focus on the Carme group, the Ananke group, and the Pasiphae group.

In the following sections, in order to use the one-sample $\mathrm{K}-\mathrm{S}$ test, three sets of observed data from Jupiter's moons will be tested against some commonly used distributions in statistics. The list of these distributions is shown in Table 1.

For the 9 continuous distributions in Table 1, the onesample K-S test will be used to select the distribution with the highest confidence level. In order to characterize these distributions with well-defined parameter values, maximum likelihood estimation is also used. In addition, the parameter values of these distributions can be calculated from the observed data. However, when confidence level (typically set to 0.05 ) decreases, the rejection domain of the test becomes smaller, so the observed values that initially fall into the rejection domain may eventually fall into the acceptance domain. This situation will bring some trouble in practical application. To this end, we adopt $p$ value, which represents the obtained confidence level by using the one-sample $\mathrm{K}$ $S$ test. In addition, the use of $p$ value not only avoids determining the level of significance in advance, but also makes it easy to draw conclusions about the test by comparing the $p$ value and significance level of the test. If the $p$ value is greater than 0.05 , we declare that the null hypothesis can be accepted. Furthermore, if the $p$ values of several distributions are all greater than 0.05 , the distribution with the largest $p$ value should be selected, and the corresponding distribution will be the most appropriate one to fit the observed data. Our results can be found in the tables in Appendix B.

\section{Distribution Inference of Satellite Groups}

In this section, distributions of several diverse physical and orbital properties for the Carme group, Ananke group, and Pasiphae group are inferred sequentially.

3.1. Carme Group. There are 15 moons in the Carme group (please see Appendix A.). Due to the lack of enough data from S/2010 J1, the number of adopted moons is 14 , which means the length of each data set is 14 from the mathematical perspective. In addition, considering all the mean densities have been calculated at being $2.60 \mathrm{~g} / \mathrm{cm}^{3}$, the surface gravity of Carme and other moons in this group is $0.017 \mathrm{~m} / \mathrm{s}^{2}$ and $0.001 \mathrm{~m} / \mathrm{s}^{2}$, respectively [19]. Therefore, these data obviously do not obey the distributions.

Based on the previous method of statistical distribution inference and MATLAB 2016a (Intel Core i5-3230 M, CPU $2.60 \mathrm{GHz}$ ), distribution inferences in the Carme group can 
TABLE 1: A list of common distributions.

\begin{tabular}{|c|c|c|}
\hline Name of Selected Distributions & Parameters & Meaning \\
\hline \multirow{2}{*}{ Beta } & $a$ & first shape parameter \\
\hline & $b$ & second shape parameter \\
\hline \multirow{2}{*}{ Birnbaum-Saunders } & $\beta$ & scale parameter \\
\hline & $\gamma$ & shape parameter \\
\hline \multirow{2}{*}{ Gamma } & $a$ & shape parameter \\
\hline & $b$ & scale parameter \\
\hline \multirow{2}{*}{ Logistic } & $\mu$ & mean \\
\hline & $\sigma$ & scale parameter \\
\hline \multirow{2}{*}{ Nakagami } & $\mu$ & shape parameter \\
\hline & $\omega$ & scale parameter \\
\hline \multirow{2}{*}{ Normal } & $\mu$ & mean \\
\hline & $\sigma$ & standard deviation \\
\hline \multirow{2}{*}{ Rice } & $s$ & noncentrality parameter \\
\hline & $\sigma$ & scale parameter \\
\hline \multirow{3}{*}{$t$ location-scale } & $\mu$ & location parameter \\
\hline & $\sigma$ & scale parameter \\
\hline & $v$ & shape parameter \\
\hline \multirow{2}{*}{ Weibull } & $A$ & scale parameter \\
\hline & $B$ & shape parameter \\
\hline
\end{tabular}

TABLE 2: The distribution inference in each physical and orbital characteristic (Carme group).

\begin{tabular}{|c|c|c|c|}
\hline Characteristics & $\begin{array}{c}\text { Distribution } \\
\text { inference }\end{array}$ & $\begin{array}{l}\text { Parameter } \\
\text { estimates }\end{array}$ & $p$-value \\
\hline $\begin{array}{l}\text { Semi-major axis } \\
\left(10^{7} \mathrm{~km}\right)\end{array}$ & $\begin{array}{c}\text { logistic } \\
\text { distribution }\end{array}$ & $\begin{array}{c}\mu=2.3326, \sigma= \\
0.00651346\end{array}$ & 0.9988 \\
\hline $\begin{array}{l}\text { Mean orbit velocity } \\
\left(10^{3} \mathrm{~km} / \mathrm{h}\right)\end{array}$ & $\begin{array}{c}\text { logistic } \\
\text { distribution }\end{array}$ & $\begin{array}{c}\mu=8.21989, \sigma= \\
0.0112805\end{array}$ & 0.6607 \\
\hline $\begin{array}{l}\text { Orbit eccentricity } \\
\left(10^{-1}\right)\end{array}$ & $\begin{array}{l}\text { Birnbaum- } \\
\text { Saunders } \\
\text { distribution }\end{array}$ & $\begin{array}{c}\beta=2.54254, \gamma= \\
0.0330888\end{array}$ & 0.6245 \\
\hline $\begin{array}{l}\text { Inclination of orbit } \\
\left(10^{2} \circ\right)\end{array}$ & $\begin{array}{l}t \text { location-scale } \\
\text { distribution }\end{array}$ & $\begin{array}{c}\mu=1.6511, \sigma= \\
0.0017, v=0.8751\end{array}$ & 0.6662 \\
\hline $\begin{array}{l}\text { Equatorial radius } \\
(\mathrm{km})\end{array}$ & $\begin{array}{c}t \text { location-scale } \\
\text { distribution }\end{array}$ & $\begin{array}{c}\mu=1.65708, \sigma= \\
0.440683, v= \\
1.14501\end{array}$ & 0.7119 \\
\hline $\begin{array}{l}\text { Escape velocity } \\
(\mathrm{km} / \mathrm{h})\end{array}$ & $\begin{array}{l}t \text { location-scale } \\
\text { distribution }\end{array}$ & $\begin{array}{c}\mu=7.32997, \sigma= \\
1.58419, v=1.06821\end{array}$ & 0.6619 \\
\hline
\end{tabular}

be found in Table 2, and the last column represents the $p$ value. The smaller the $p$ value, the greater the significance because it tells us that hypothesis under consideration may not be sufficient to explain the observations. The hypothesis will be rejected if any of these probabilities is less than or equal to a small, fixed but arbitrarily predefined threshold value. The null hypothesis here refers to data obeying a particular distribution, and the alternative hypothesis assumes that the data does not obey the distribution.

From Table 2, semi-major axis and mean orbit velocity obey the logistic distribution, of which parameter $\mu$ denotes the average orbital eccentricity and parameter $\sigma$ plays a key role in representing the variance of the data set, through the variance formula $\sigma^{2} \pi^{2} / 3$, so the variances of these logistic distributions are 1.3957E-04 and 4.1863E-04, respectively. As the parameter $\mu$ increases, it indicates that the average semi-major axis and mean orbit velocity of the Carme group increase. As the parameter $\sigma$ increases, the data in these two characteristics gradually disperse, and the discrepancy between the data and the average value increases. The meaning of decreasing the parameter $\mu$ and $\sigma$ shares the same principle as that of increasing the parameters. The orbit eccentricity follows the Birnbaum-Saunders distribution, which is unimodal with a median of $\beta$. The mean value and the variance of the distribution can be calculated by the following relationships:

$$
\mu=\beta\left(1+\frac{\gamma^{2}}{2}\right),
$$


TABLE 3: The distribution inference in each orbital or physical characteristic (Ananke group).

\begin{tabular}{|c|c|c|c|}
\hline Characteristics & $\begin{array}{c}\text { Distribution } \\
\text { inference }\end{array}$ & $\begin{array}{l}\text { Parameter } \\
\text { estimates }\end{array}$ & $p$-value \\
\hline $\begin{array}{l}\text { Semi-major axis } \\
\left(10^{7} \mathrm{~km}\right)\end{array}$ & $\begin{array}{l}t \text { location-scale } \\
\text { distribution }\end{array}$ & $\begin{array}{c}\mu=2.11665, \sigma= \\
0.00915557, v= \\
1.52402\end{array}$ & 0.9304 \\
\hline $\begin{array}{l}\text { Mean orbit velocity } \\
\left(10^{3} \mathrm{~km} / \mathrm{h}\right)\end{array}$ & $\begin{array}{c}\text { Weibull } \\
\text { distribution }\end{array}$ & $\begin{array}{c}A=2.29923, B= \\
21.9834\end{array}$ & 0.9788 \\
\hline $\begin{array}{l}\text { Orbit eccentricity } \\
\left(10^{-1}\right)\end{array}$ & $\begin{array}{l}t \text { location-scale } \\
\text { distribution }\end{array}$ & $\begin{array}{c}\mu=8.72481, \sigma= \\
0.0203158, v= \\
1.36328\end{array}$ & 0.6017 \\
\hline $\begin{array}{l}\text { Inclination of orbit } \\
\left(10^{2} \circ\right)\end{array}$ & $\begin{array}{l}t \text { location-scale } \\
\text { distribution }\end{array}$ & $\begin{array}{c}\mu=1.4879, \sigma= \\
0.0127, v=1.4538\end{array}$ & 0.9075 \\
\hline $\begin{array}{l}\text { Equatorial radius } \\
(\mathrm{km})\end{array}$ & $\begin{array}{l}t \text { location-scale } \\
\text { distribution }\end{array}$ & $\begin{array}{c}\mu=1.80724, \sigma= \\
0.660297, v= \\
1.19615\end{array}$ & 0.7231 \\
\hline Mass $\left(10^{13} \mathrm{~kg}\right)$ & $\begin{array}{c}\text { Weibull } \\
\text { distribution }\end{array}$ & $\begin{array}{c}A=38.3573, B= \\
0.368827\end{array}$ & 0.4304 \\
\hline $\begin{array}{l}\text { Surface gravity } \\
\left(\left(\mathrm{m} / \mathrm{s}^{2}\right) / 10^{3}\right)\end{array}$ & $\begin{array}{l}t \text { location-scale } \\
\text { distribution }\end{array}$ & $\begin{array}{c}\mu=0.167044, \sigma= \\
0.0636087, v= \\
1.40815\end{array}$ & 0.4210 \\
\hline $\begin{array}{l}\text { Escape velocity } \\
(\mathrm{km} / \mathrm{h})\end{array}$ & $\begin{array}{l}t \text { location-scale } \\
\text { distribution }\end{array}$ & $\begin{array}{c}\mu=8.24903, \sigma= \\
2.61085, v=1.15837\end{array}$ & 0.7262 \\
\hline
\end{tabular}

$$
\sigma^{2}=(\beta \gamma)^{2}\left(1+\frac{5 \gamma^{2}}{4}\right)
$$

Therefore, the mean value of orbital eccentricity can be calculated to be 0.2543 and the variance is 7.08E-9.

The inclination of orbit, equatorial radius, and escape velocity are subject to the $t$ location-scale distribution, which contains the scale parameter $\sigma$, the location parameter $\mu$, and the shape parameter $v$. Without loss of generality, we assume that the data vector $x$ obeys the $t$ location-scale distribution, and then we have $(x-\mu) / \sigma \sim t(v)$, which obeys Student's $t$-distribution; here $v$ represents the degrees of freedom. As can also be seen in Table 2, the inclination of orbit obeys the $t$ location-scale distribution with parameters $(1.6511,0.0017,0.8751)$ and the mean inclination is $165.11^{\circ}$. The equatorial radius follows the $t$ location-scale distribution with parameters $(1.65708,0.440683,1.14501)$. Therefore, the moons in Carme group have an average equatorial radius of 1.65708 $\mathrm{km}$. In addition, the escape velocity characteristic obeys the $t$ location-scale distribution with parameters $(7.32997,1.58419$, 1.06821 ) and the average escape velocity is $7.32997 \mathrm{~km} / \mathrm{h}$. As the parameter $\mu$ changes, the average equatorial radius and escape velocity of the group also change accordingly. In the $t$ location-scale distribution, the variance is $\sigma^{2} v /(v-2)$, and when the shape parameter $v$ is greater than two, the variance of the distribution is defined. Therefore, the variances of these two specific $t$ location-scale distributions cannot be defined.

3.2. Ananke Group. There are 10 moons in the Ananke group, of which we do not have enough data about S/2010 J2. Thus, the length of each characteristic of the remaining moons in this group will be 9 .
From our discussion on the $t$ location-scale distribution in Carme group, it is easy to understand the distribution inference of semi-major axis, mean orbit velocity, equatorial radius, surface gravity, and escape velocity in the Ananke group (see Table 3). However, compared with Table 2, it is noted that the orbit eccentricity and mass properties in the Ananke group are subject to the Weibull distribution. Parameters $A$ and $B$ represent the scale and shape parameters of the distribution [20], respectively, which together determine the mean and variance of the distribution.

3.3. Pasiphae Group. There are 19 moons in the Pasiphae group; due to the lack of data about three moons, S/2011 J2, S/2017 J1, and S/2016 J1, the remaining 16 will be studied in this subsection.

The distributions in Table 4 are inferred to be similar to the distribution in Tables 2 and 3. Therefore, we can easily understand the parameters of these distributions.

\section{Comparison of Data Properties}

Table 5 is given according to the previous distribution inference.

Based on the previous distribution inference, the properties of the moons' data can be compared more specifically and conveniently. According to the distribution of the specific parameters, we get the following probability density function (PDF) diagram.

As shown in Figure 1, the semi-major axis in the Ananke group is the smallest, followed by the Carme group and the Pasiphae group. In addition, the PDF of the Pasiphae group is relatively flat, indicating a large dispersion in semi-major axis around Jupiter, while the data in the Carme and Ananke groups differ slightly. As can be seen from Figure 2, the 
TABLE 4: The distribution inference in each orbital or physical characteristic (Pasiphae group).

\begin{tabular}{lccc}
\hline Characteristics & Distribution inference & Parameter estimates & $p$-value \\
\hline Semi-major axis $\left(10^{7} \mathrm{~km}\right)$ & $t$ location-scale distribution & $\mu=2.38241, \sigma=0.0273627, v=0.730397$ & 0.3730 \\
Mean orbit velocity $\left(10^{3} \mathrm{~km} / \mathrm{h}\right)$ & logistic distribution & $\mu=8.22803, \sigma=0.226046$ & 0.4015 \\
Orbit eccentricity $\left(10^{-1}\right)$ & logistic distribution & $\mu=2.95251, \sigma=0.599373$ & 0.9550 \\
Inclination of orbit $\left(10^{2} \circ\right)$ & logistic distribution & $\mu=1.5136, \sigma=0.0339$ & 0.8987 \\
Equatorial radius $(\mathrm{km})$ & Weibull distribution & $A=3.69781, B=0.781648$ & 0.0859 \\
Mass $\left(10^{13} \mathrm{~kg}\right)$ & Weibull distribution & $A=72.5121, B=0.266585$ & 0.1096 \\
Surface gravity $\left(\left(\mathrm{m} / \mathrm{s}^{2}\right) / 10^{3}\right)$ & Weibull distribution & $A=3.09876, B=0.843794$ & 0.0594 \\
Escape velocity $(\mathrm{km} / \mathrm{h})$ & Weibull distribution & $A=17.0302, B=0.807236$ & 0.0722 \\
\hline
\end{tabular}

TABLE 5: Distribution inference summary.

\begin{tabular}{lccc}
\hline Characteristics & Carme Group & Ananke Group & Pasiphae Group \\
\hline Semi-major axis & logistic distribution & $t$ location-scale distribution & $t$ location-scale distribution \\
Mean orbit velocity & logistic distribution & $t$ location-scale distribution & logistic distribution \\
Orbit eccentricity & Birnbaum-Saunders distribution & Weibull distribution & logistic distribution \\
Inclination of orbit & $t$ location-scale distribution & $t$ location-scale distribution & logistic distribution \\
Equatorial radius & $t$ location-scale distribution & $t$ location-scale distribution & Weibull distribution \\
Mass & None & Weibull distribution & Weibull distribution \\
Surface gravity & None & $t$ location-scale distribution & Weibull distribution \\
Escape velocity & $t$ location-scale distribution & $t$ location-scale distribution & Weibull distribution \\
\hline
\end{tabular}

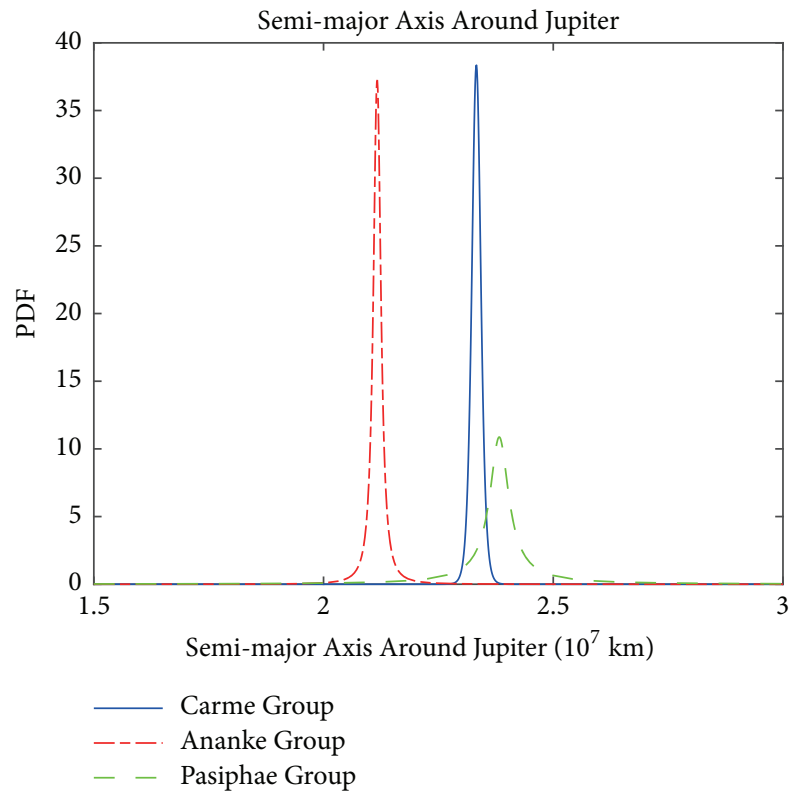

FIGURE 1: The PDF curves of semi-major axis around Jupiter.

trend of mean orbital velocity distribution in Carme group is the closest to each other, with the mean value being the smallest among the three groups, followed with the Pasiphae and Ananke groups. Obviously, the curve of the Pasiphae group is the flattest, which shows the mean orbit velocity in the Pasiphae group differing greatly. Figure 3 shows that the mean value in the Ananke group is the smallest, followed by the Carme and Pasiphae groups. It is obvious that the PDF curve of the Pasiphae group is flatter compared to the others,

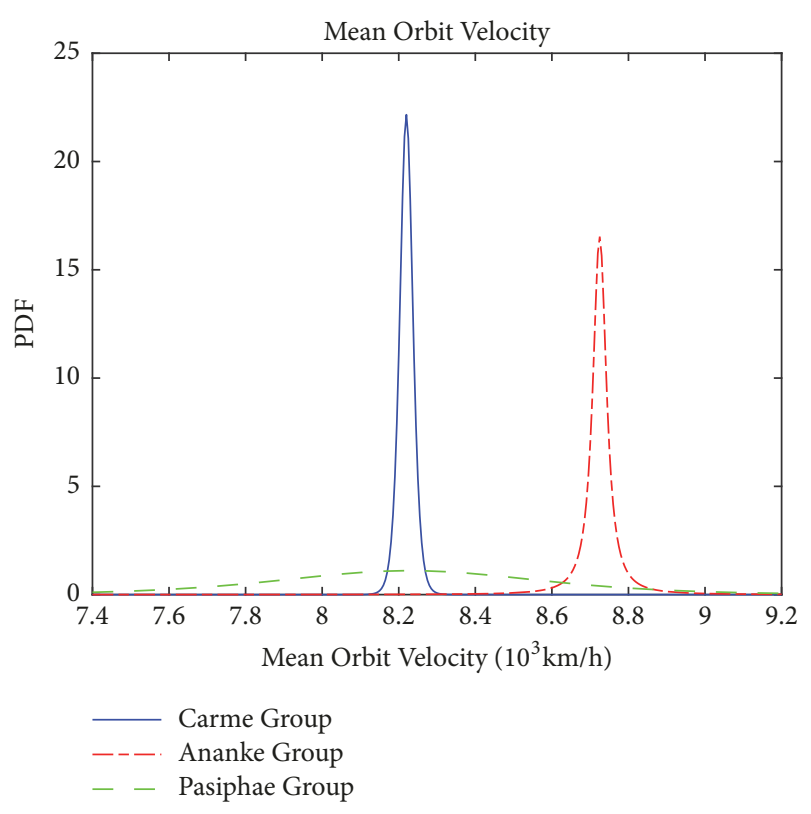

FIgURE 2: The PDF curves of mean orbit velocity.

which means the orbital eccentricity of the Pasiphae group has a relatively large dispersion. In Figure 4, although the inclinations of orbit in the Ananke group and Pasiphae group are relatively close in value compared with the Carme group, and the data looks more dispersed than those in Carme group, the inclination of orbit in the Ananke group has the same distribution as the Carme group.

Figure 5 shows the PDF curves of equatorial radii of these three groups. Data attributes are similar; most of the moons' 


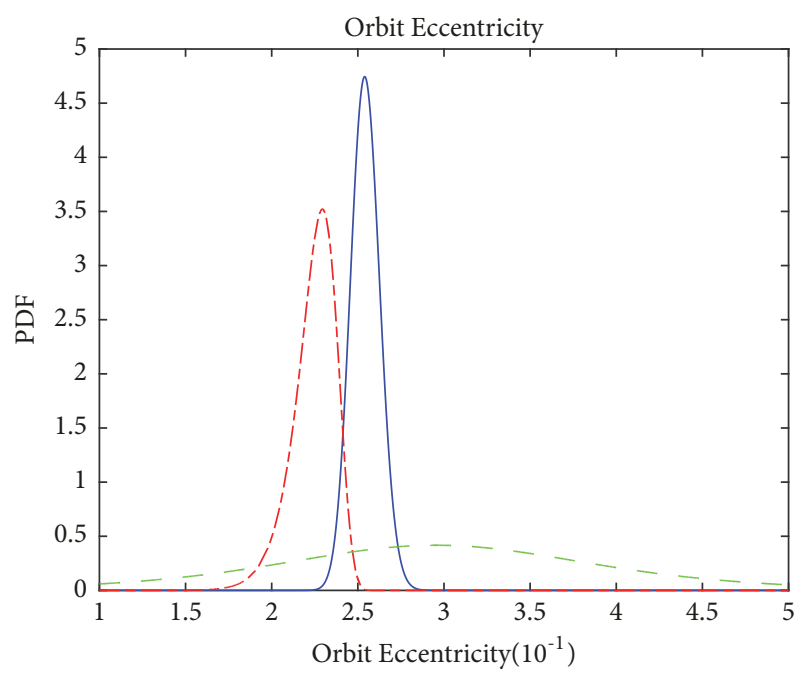

-- Ananke Group
$-\quad$ Pasiphae Group

Figure 3: The PDF curves of orbit eccentricity.

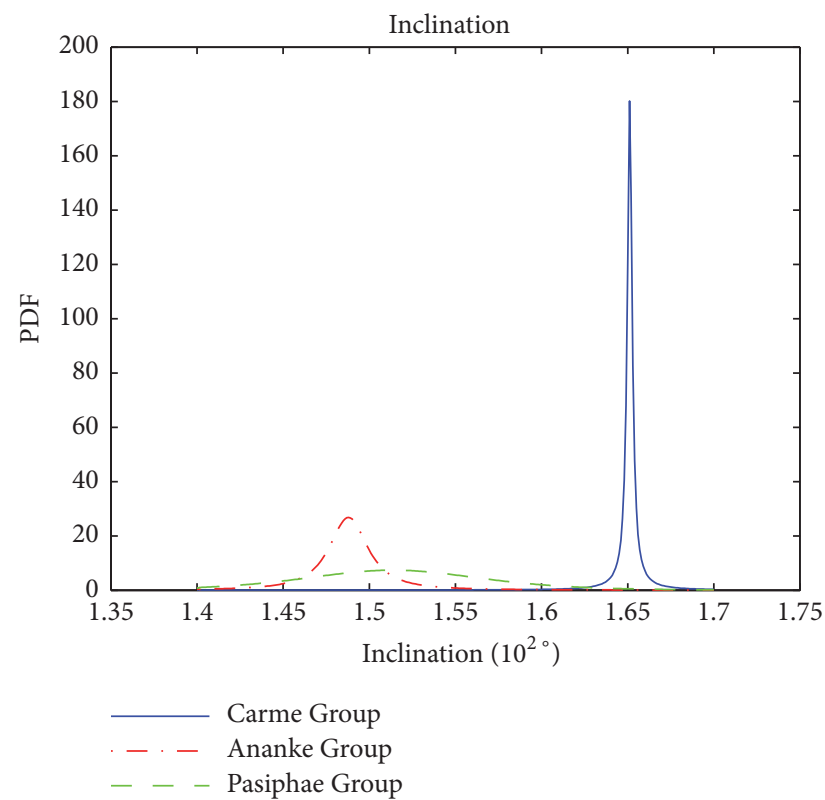

FIgURE 4: The PDF curves of inclination.

radiuses are less than $4 \mathrm{~km}$. Figure 6 indicates similarities between the Ananke and Pasiphae groups. As can be seen from these curves, most of the moons in these two groups are of relatively small mass.

Figure 7 illustrates the surface gravity PDF curves in the Ananke and Pasiphae groups. The difference in density between the two groups indicates that the surface gravity of the Ananke moons is higher than that of the Pasiphae group. The PDF plots corresponding to the escape velocity are shown in Figure 8, where it is clear that the Pasiphae group is flatter than the other two groups. The Carme group also has similar

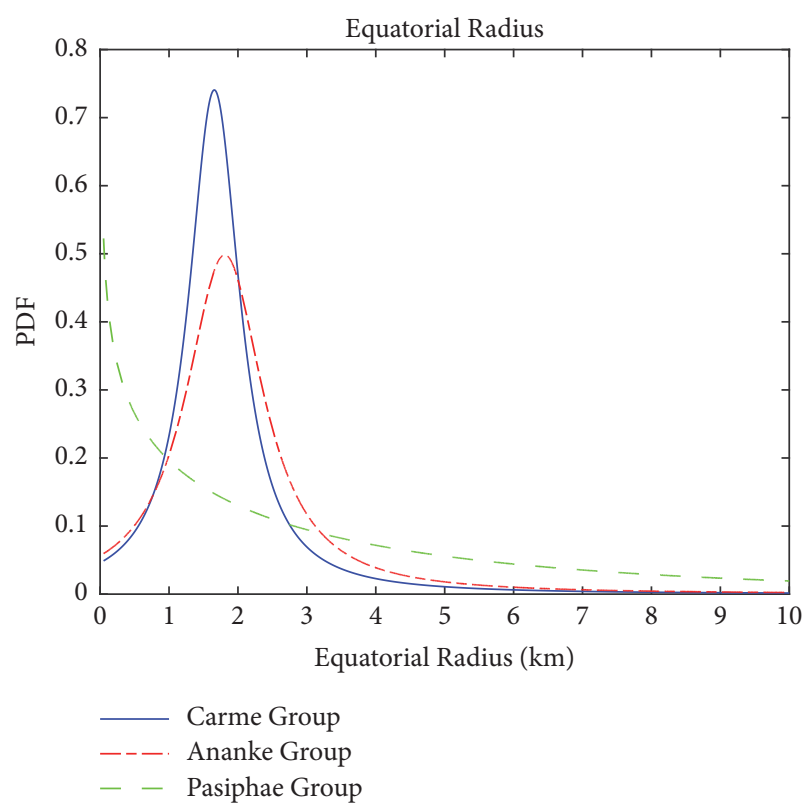

FIgURE 5: The PDF curves of equatorial radius.

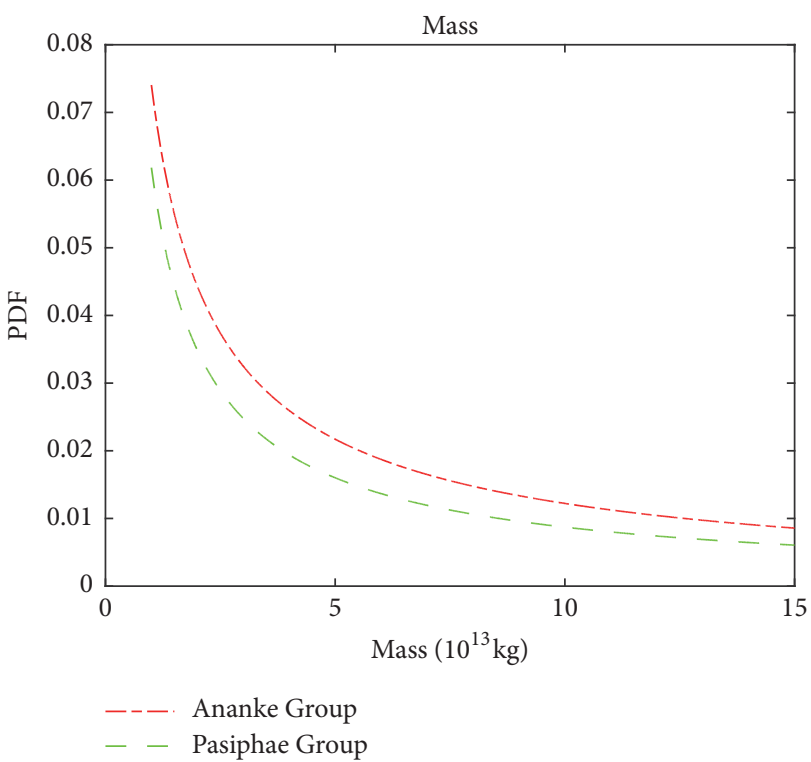

FIGURE 6: The PDF curves of mass.

escape velocity density to the Ananke group, but the former average escape velocity is smaller.

\section{Verification of Rationality of Theoretical Results}

In this section, we take the semi-major axis and mean orbit velocity of the moons in the Carme group as concrete examples to illustrate the rationality of the statistical inferences in the preceding sections. As can be seen in Table 2, semimajor axis (sma) and mean orbit velocity ( $m o v)$ obey the logistic distribution with parameters $(2.3326,0.00651346)$ 


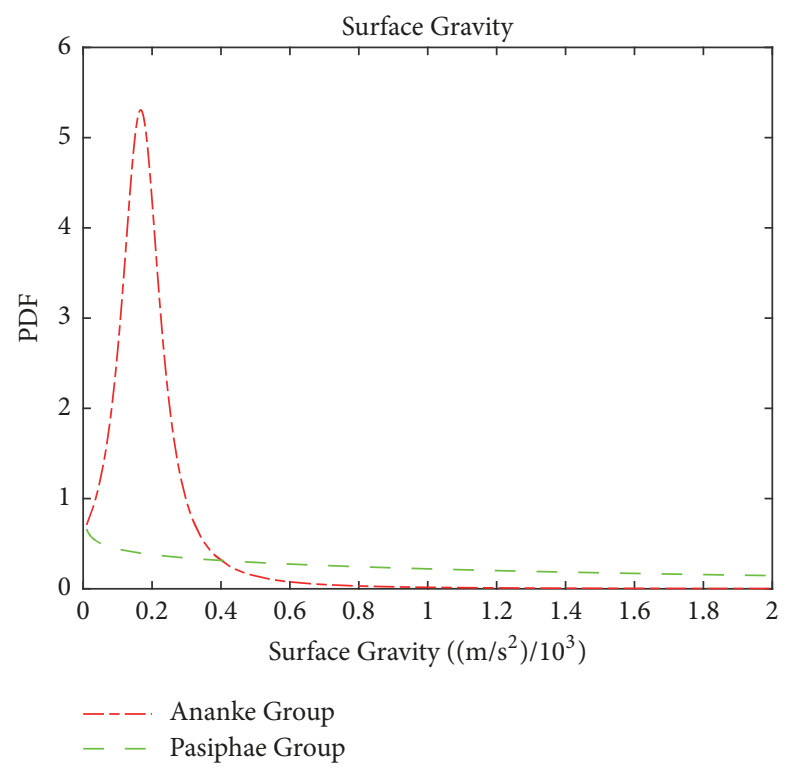

Figure 7: The PDF curves of surface gravity.

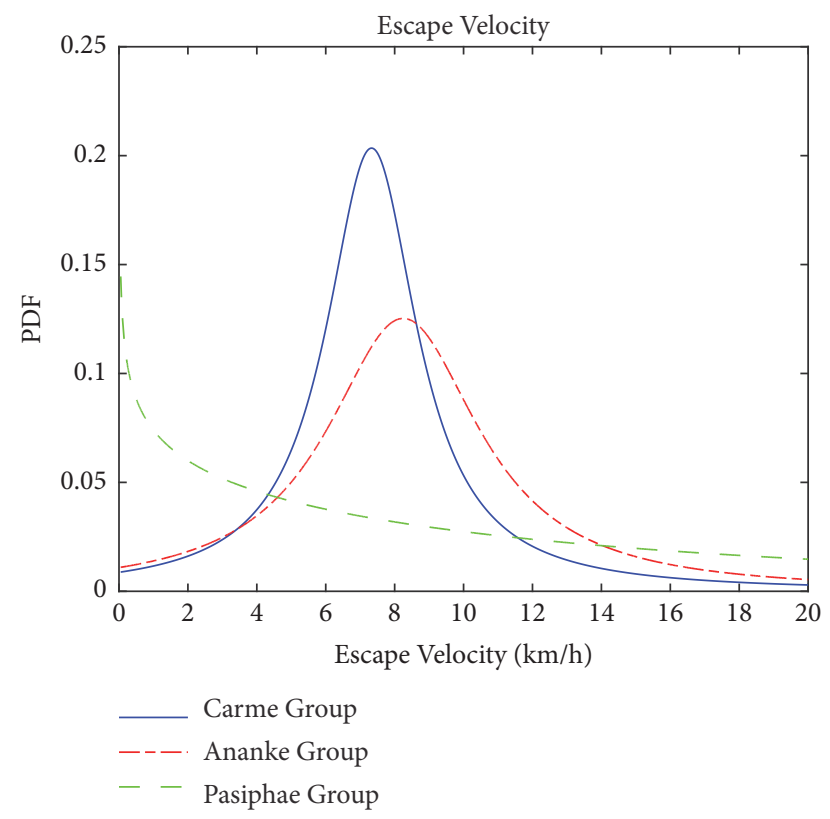

FIGURE 8: The PDF curves of escape velocity.

and $(8.21989,0.0112805)$, respectively. So the corresponding predicted PDFs can be written as

$$
\begin{aligned}
& f_{\text {pre }, \text { sma }}(v ; \mu, \sigma) \\
& \quad=\frac{e^{-(v-2.3326) / 0.00651346}}{0.00651346\left(1+e^{-(v-2.3326) / 0.00651346}\right)^{2}}
\end{aligned}
$$

and

$$
\begin{aligned}
& f_{\text {pre }, \text { mov }}(v ; \mu, \sigma) \\
& \quad=\frac{e^{-(v-8.21989) / 0.00112805}}{0.00112805\left(1+e^{-(v-8.21989) / 0.00112805}\right)^{2}} .
\end{aligned}
$$

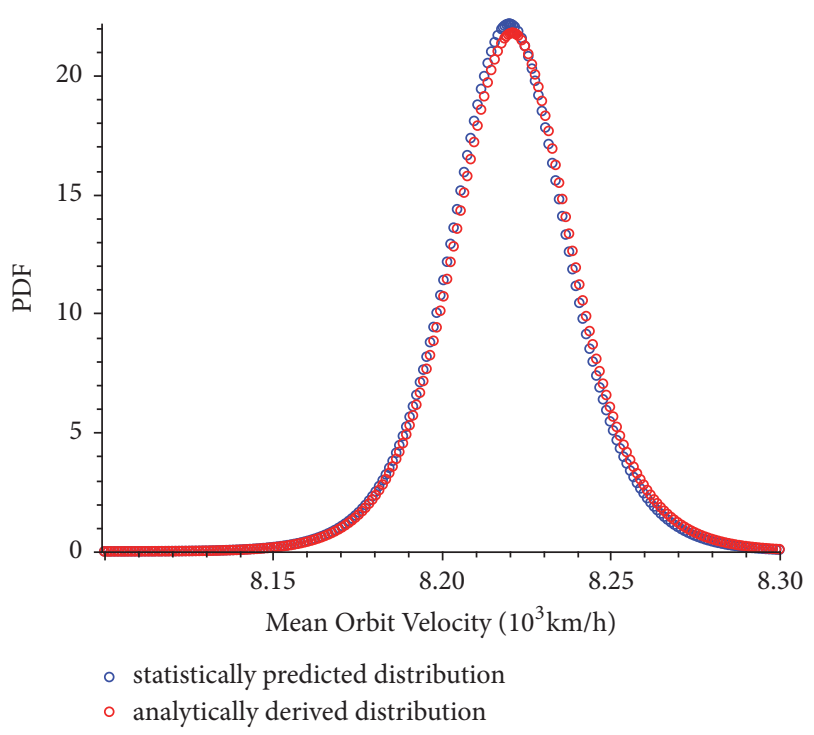

FIgURE 9: Comparison of statistical prediction distribution and analytical derivation distribution.

Note that the orbital and physical properties are not independent of each other. For instance, the semi-major axis $r$ is related to the mean orbit velocity $v$ through the relation $v=\sqrt{G M / r}$, where $G M$ is the mass parameter. Then the PDF of mean orbit velocity can also be derived analytically as follows

$$
\begin{aligned}
& f_{\text {ana }, \text { mov }}(v ; \mu, \sigma) \\
& \quad=2 \frac{G M}{v^{3}} f_{\text {pre }, \text { sma }}\left(\frac{G M}{v^{2}} ; 2.3326,0.00651346\right) \\
& \quad=2 \frac{G M}{v^{3}} \frac{e^{-\left(G M-2.3326 v^{2}\right) / 0.00651346 v^{2}}}{0.00651346\left(1+e^{\left.-\left(G M-2.3326 v^{2}\right) / 0.00651346 v^{2}\right)^{2}}\right.}
\end{aligned}
$$

Although the PDF of mean orbit velocity obtained by different methods has different mathematical representations, from Figure 9, we can find that $f_{\text {ana,mov }}(v ; \mu, \sigma)$ (PDF curve represented by red circles) obtained by the analytical method is in good agreement with $f_{\text {pre,mov }}(v ; \mu, \sigma)$ (PDF curve represented by blue circles) obtained by statistical inference.

However, what we need to pay attention to here is that some physical features and orbital elements are mixed and can be linked by some mathematical formulas similar to the above. Theoretically, the distribution of another variable in the formula can be solved by a known defined distribution; although this may be a complex process because the probability density function may contain some transcendental functions and gamma functions, it can be achieved. Yet the known distribution becomes uncertain now; that is, the distribution exists with a certain probability. So, there will be a certain risk when we calculate the distribution of the linked variable based on this uncertain distribution, especially when the possibility of the inferred distribution is not very high.

In addition, in order to further show that the results of the KS test agree well with the actual observed results, 
TABLE 6: Distribution inference of Carme group without Erinome (S/2000 J4).

\begin{tabular}{|c|c|c|c|}
\hline & $p$-value & $h$ & Parameter Values \\
\hline \multirow{2}{*}{ Beta } & \multirow{2}{*}{$4.02 \mathrm{E}-05$} & \multirow{2}{*}{1} & $a=0.202265$ \\
\hline & & & $b=0.173517$ \\
\hline \multirow{2}{*}{ Birnbaum-Saunders } & \multirow{2}{*}{0.0307} & \multirow{2}{*}{1} & $\beta=2.37288$ \\
\hline & & & $\gamma=0.943409$ \\
\hline \multirow{2}{*}{ Gamma } & \multirow{2}{*}{0.0308} & \multirow{2}{*}{1} & $a=1.05985$ \\
\hline & & & $b=3.11366$ \\
\hline \multirow{2}{*}{ Logistic } & \multirow{2}{*}{0.0463} & \multirow{2}{*}{1} & $\mu=1.95282$ \\
\hline & & & $\sigma=1.72324$ \\
\hline \multirow{2}{*}{ Nakagami } & \multirow{2}{*}{0.0039} & \multirow{2}{*}{1} & $\mu=0.282272$ \\
\hline & & & $\omega=43.5177$ \\
\hline \multirow{2}{*}{ Normal } & \multirow{2}{*}{0.0037} & \multirow{2}{*}{1} & $\mu=3.3$ \\
\hline & & & $\sigma=5.94531$ \\
\hline \multirow{2}{*}{ Rice } & \multirow{2}{*}{$5.73 \mathrm{E}-08$} & \multirow{2}{*}{1} & $s=0.138623$ \\
\hline & & & $\sigma=4.66385$ \\
\hline \multirow{3}{*}{$t$ location-scale } & \multirow{3}{*}{0.6806} & \multirow{3}{*}{0} & $\mu=1.67257$ \\
\hline & & & $\sigma=0.487941$ \\
\hline & & & $v=1.15623$ \\
\hline \multirow{2}{*}{ Weibull } & \multirow{2}{*}{0.0698} & \multirow{2}{*}{0} & $A=3.06061$ \\
\hline & & & $B=0.897697$ \\
\hline
\end{tabular}

Note: $h=0$ and $h=1$ indicate acceptance of the null hypothesis and rejection of the null hypothesis, respectively.

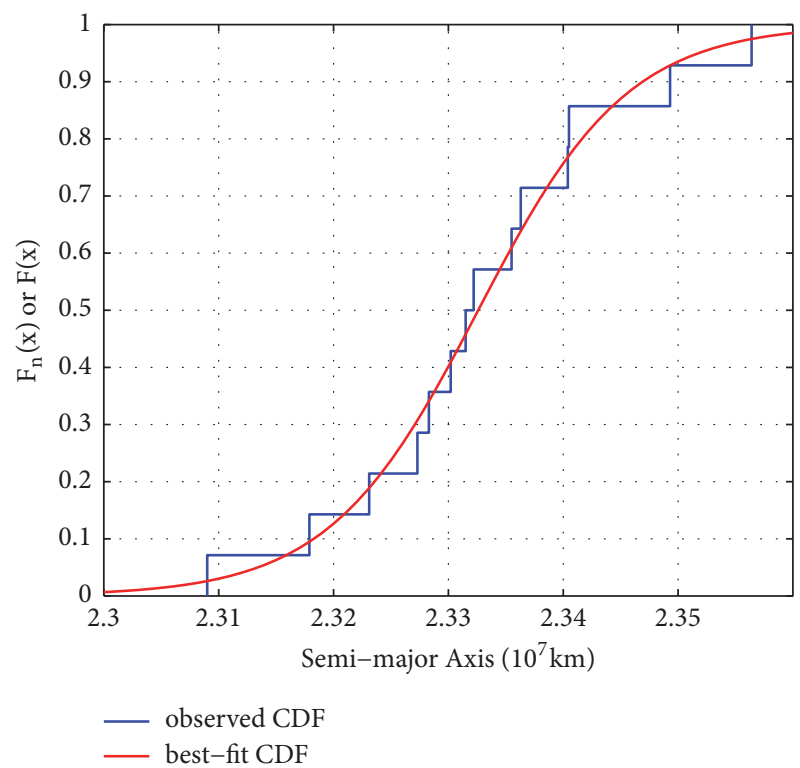

FIGURE 10: Comparison of the observed CDF and the best-fit CDF of semi-major axis.

we also compared the best-fit CDFs (the CDFs of inferred statistically) and the observed CDFs. From Figures 10 and 11, it can be seen that the best-fit CDF and the observed CDF of semi-major axis agree better than the case of mean orbital velocity. This should be due to the fact that the $p$ value of the former is 0.9988 , which is obviously larger than that of the latter 0.6607 .

\section{Application of the Distribution Inference}

As mentioned previously, identification of irregular satellite families is tentative. However, after the distribution was inferred, it is found that these features of moons in the three different groups obey some selected distributions. Furthermore, the obtained population distribution can also be used to predict the characteristic data. Considering the rationality of proving this prediction method, suppose some characteristic data of a moon is unknown in a given irregular moon group. One specific feature of the moon can be predicted by using other moons' data and one-sample K-S method. Here is an example:

Assume that some characteristic data of the moon Erinome (S/2000 J4) in Carme group is poorly known. Now, we try to predict the equatorial radius of the Erinome. First, we use one-sample K-S method to find the most appropriate continuous distribution for these data. The inference results are as shown in Table 6.

From the $p$ values displayed in Table 6 , it becomes clear that the corresponding $p$ value 0.6806 is the largest and $h=0$, so the best distribution for the remaining characteristic data in the Carme group is $t$ location-scale distribution with the PDF

$$
\begin{aligned}
& f(x \mid \mu, \sigma, v) \\
& =\frac{\Gamma((v+1) / 2)}{\sigma \sqrt{\pi v} \Gamma(v / 2)}\left[\frac{v+((x-\mu) / \sigma)^{2}}{v}\right]^{-(v+1) / 2}, \\
& -\infty<x<+\infty,
\end{aligned}
$$

where $\Gamma(\cdot)$ is the Gamma function. 


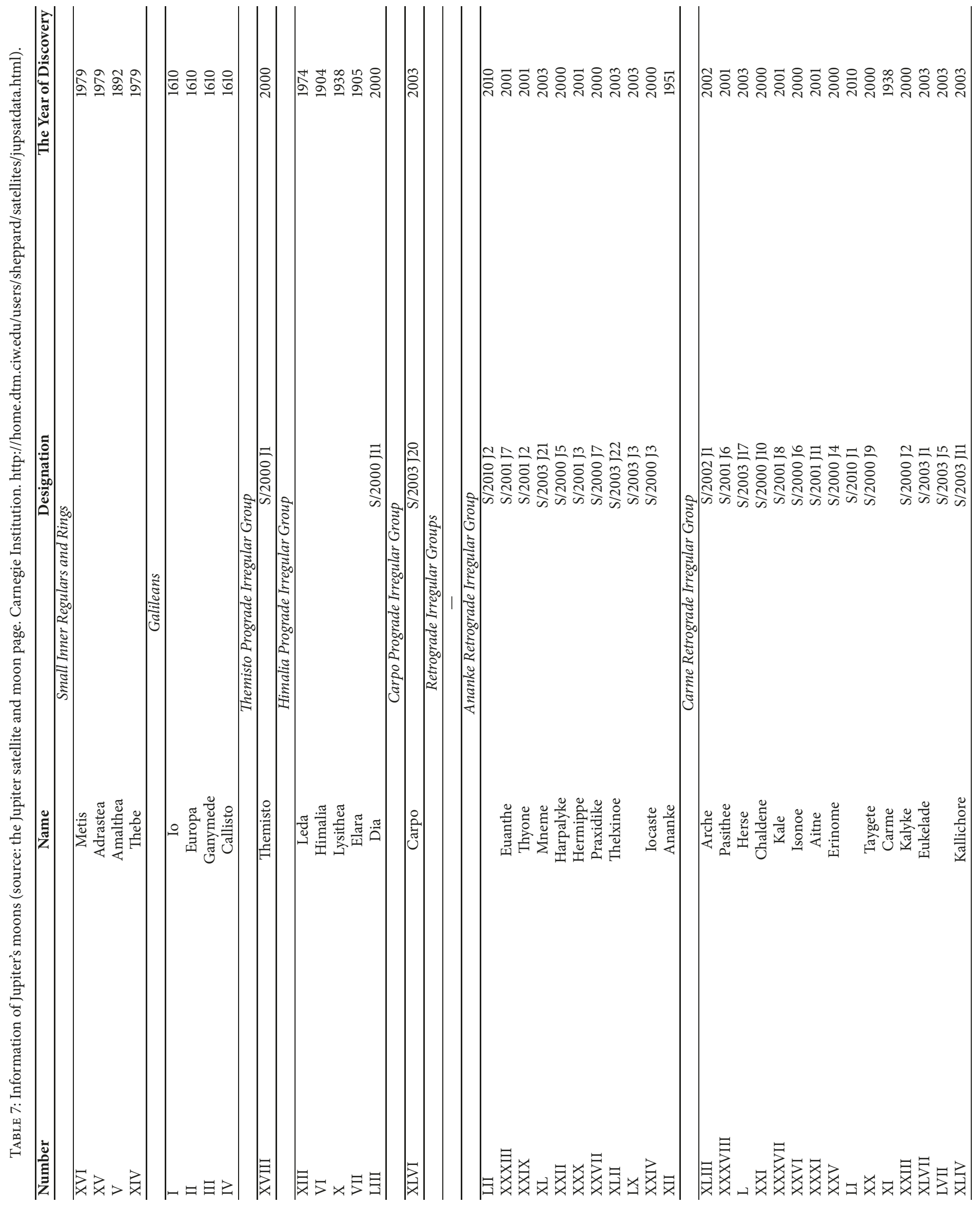




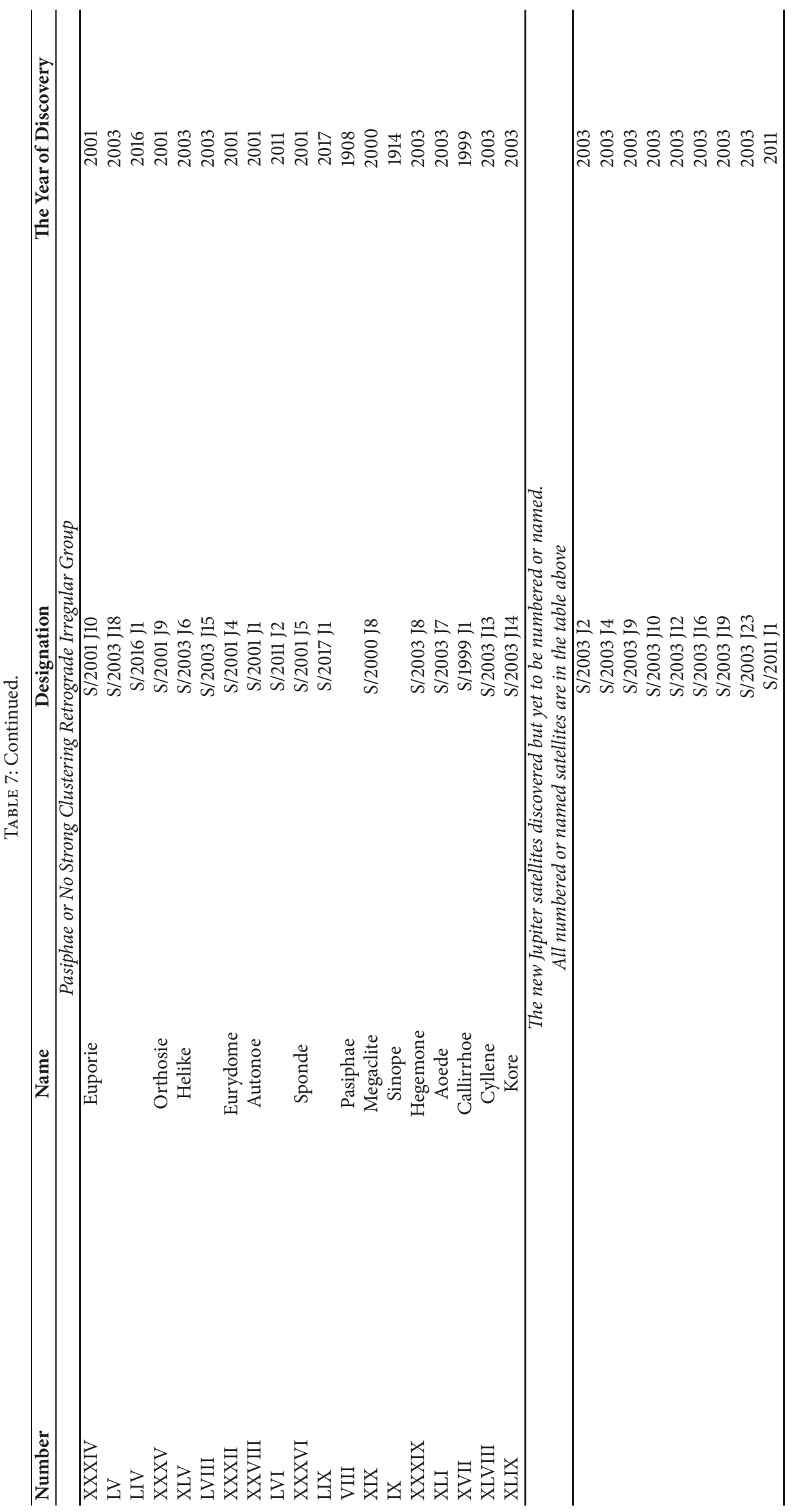




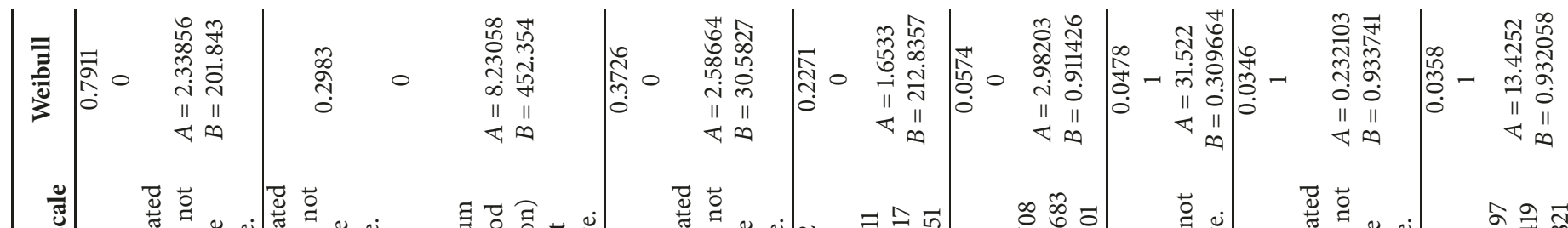

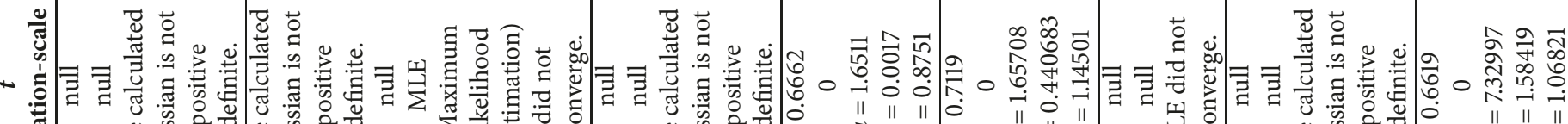

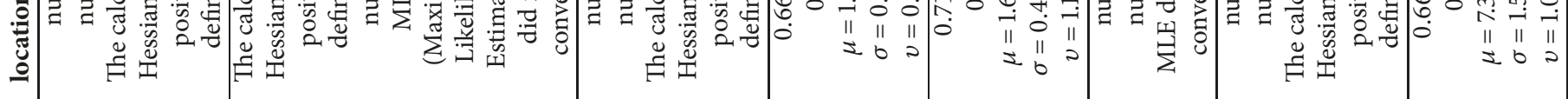

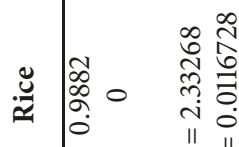

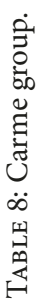

है

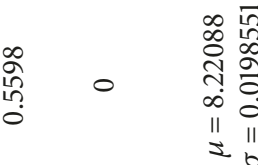

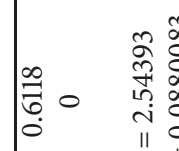

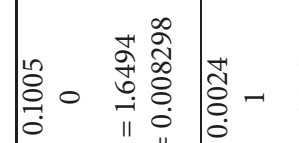

会

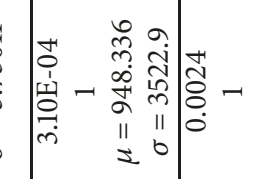

众

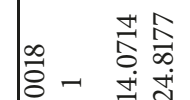

mint

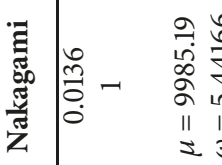

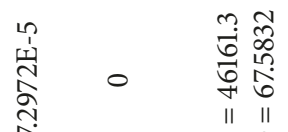

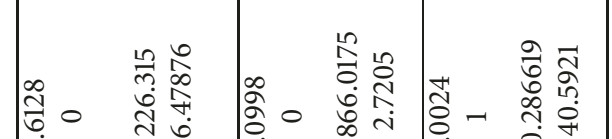

+ ลूे

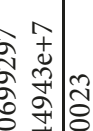

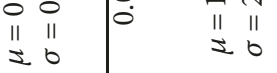

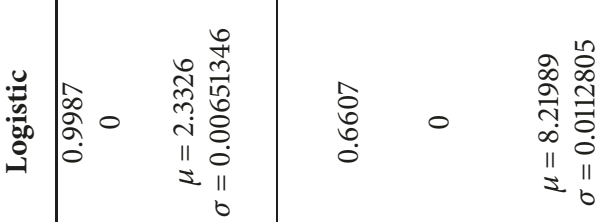

范

$=\quad \frac{\mathscr{O}}{U}$

曾

至

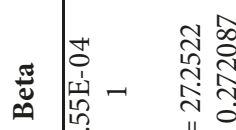

$\exists \quad \exists \quad$ 范离

|

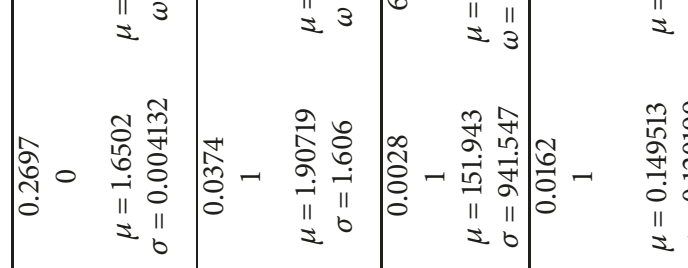

त) $\quad$ i

ठิ-

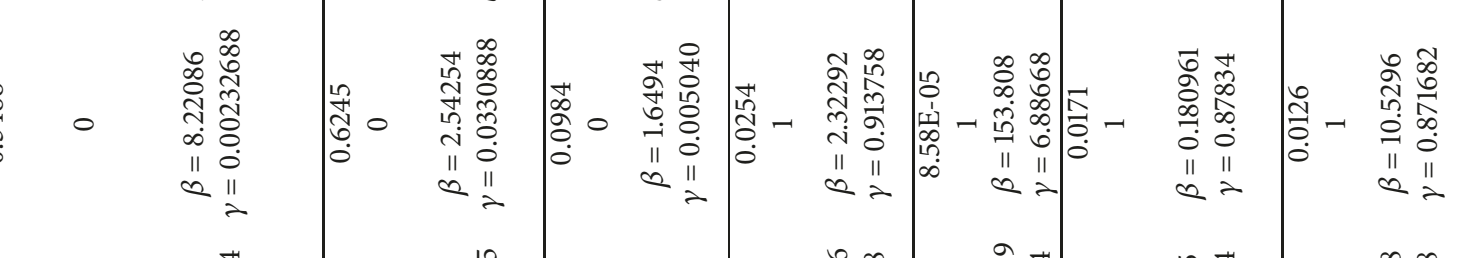

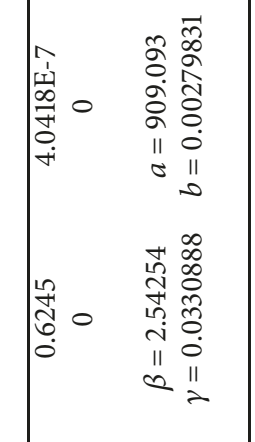

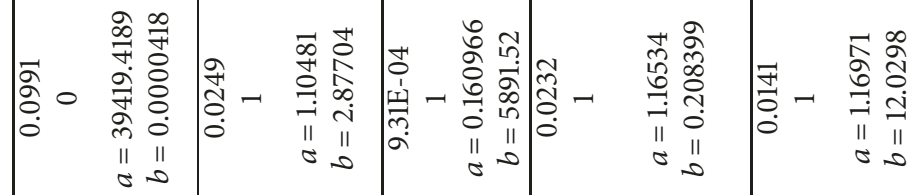

in $\quad 0$ II

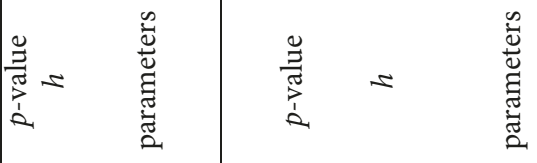

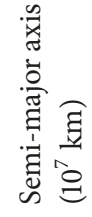

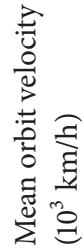

总

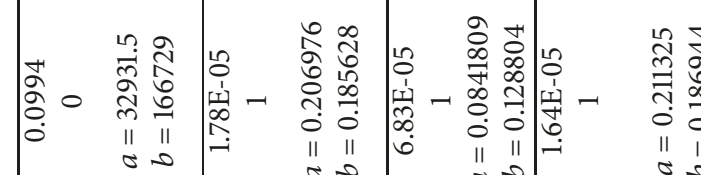

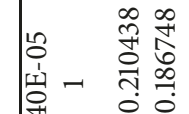

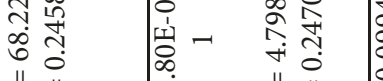




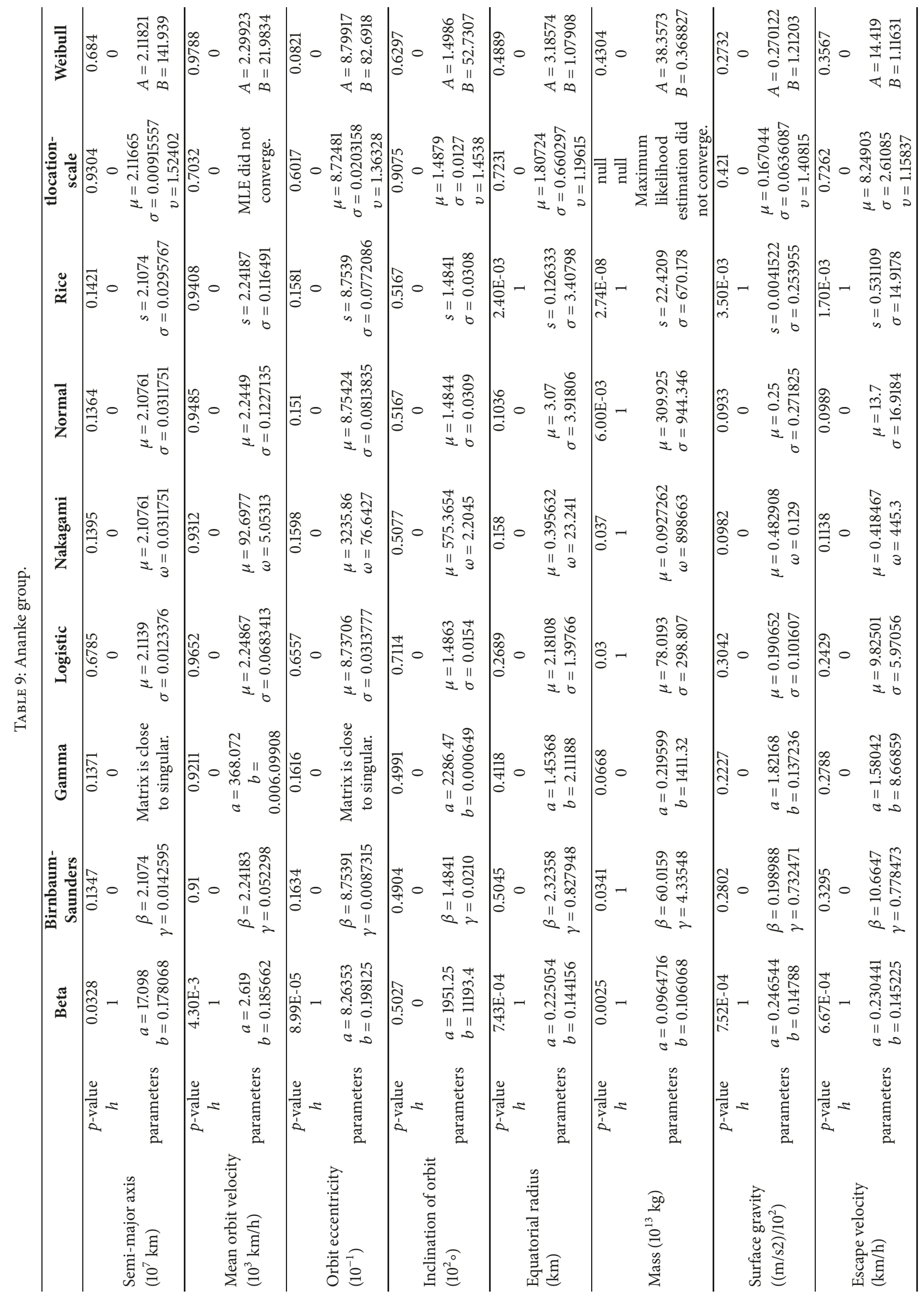




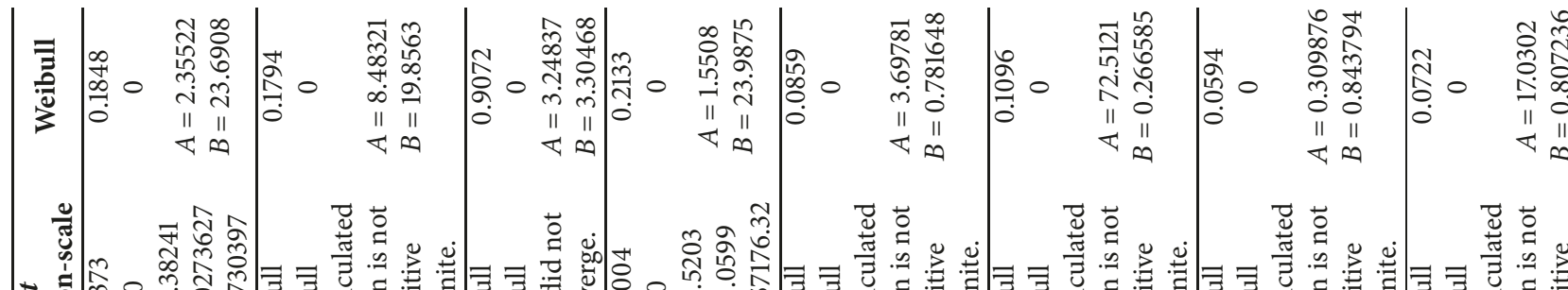

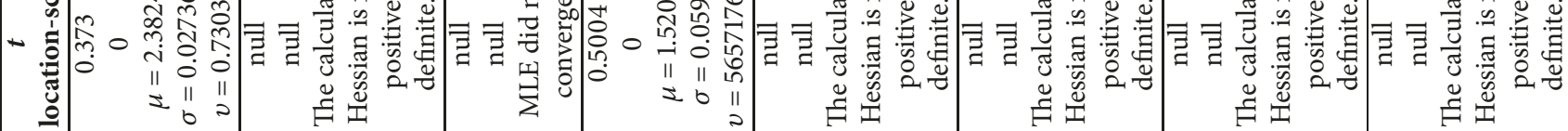

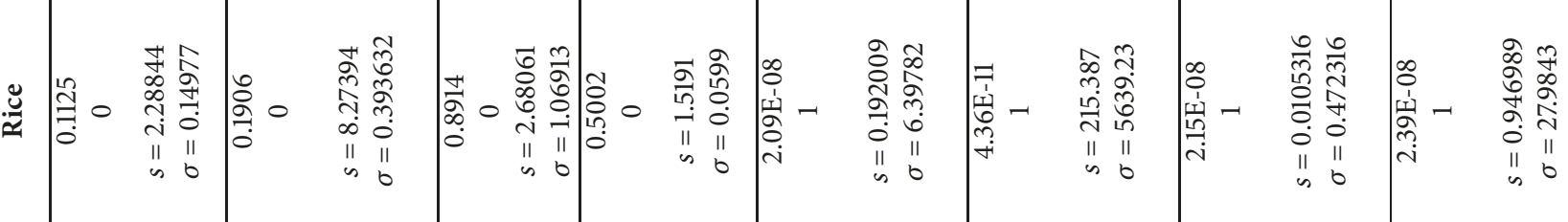

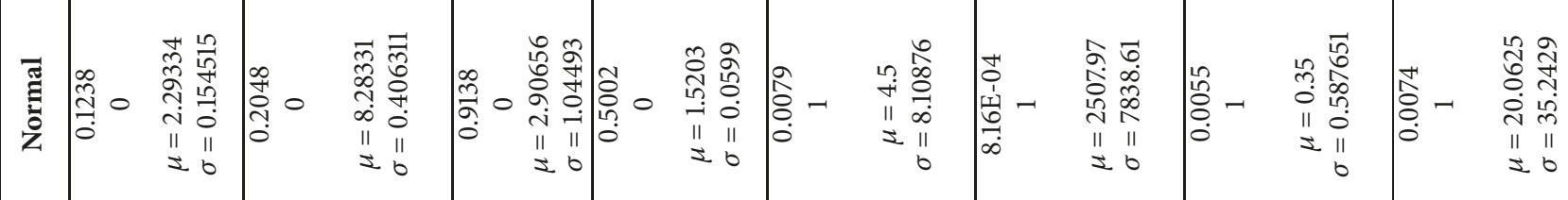

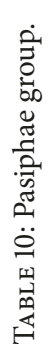

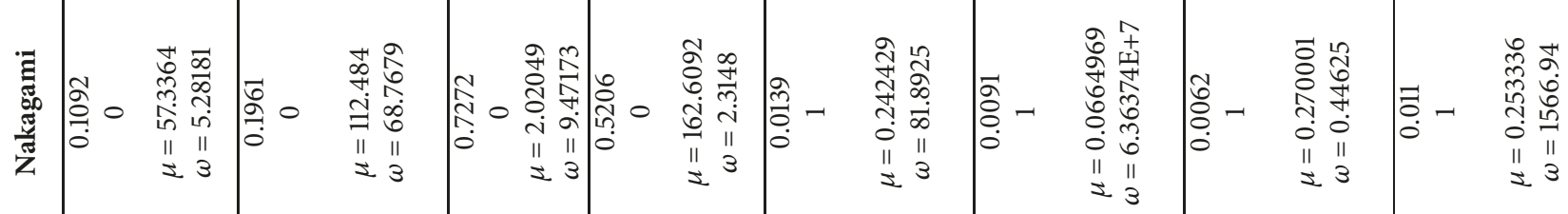

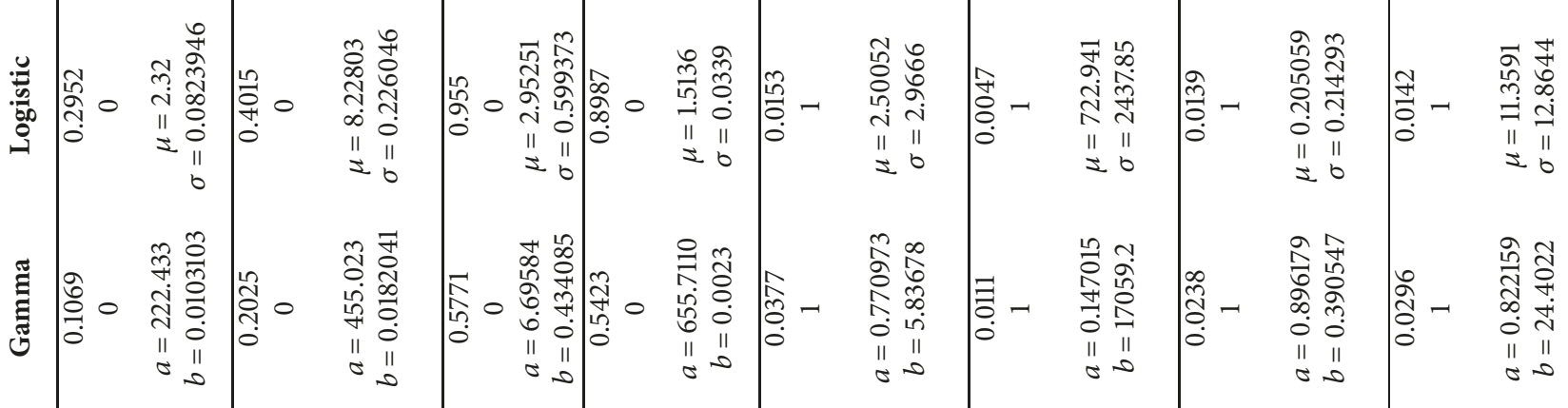

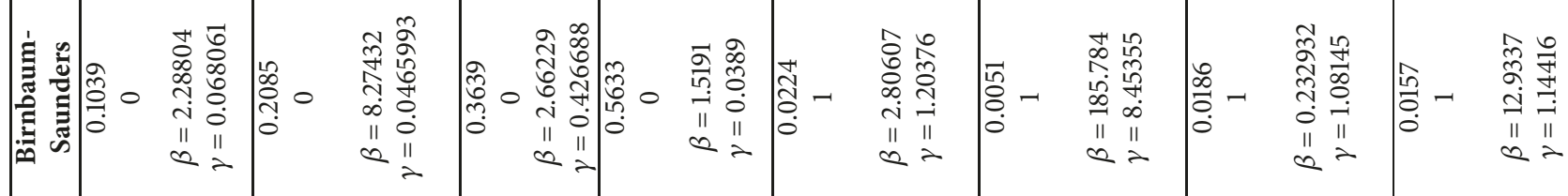

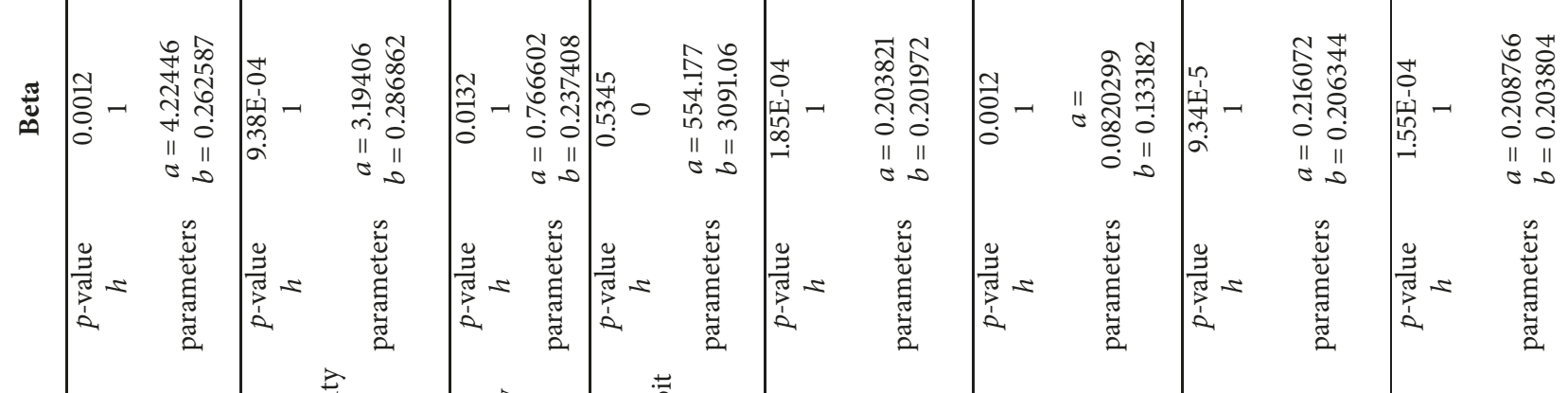

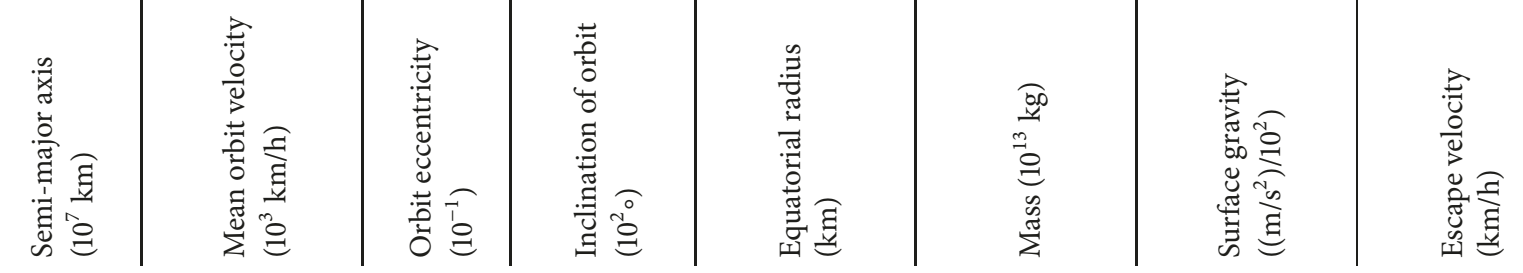




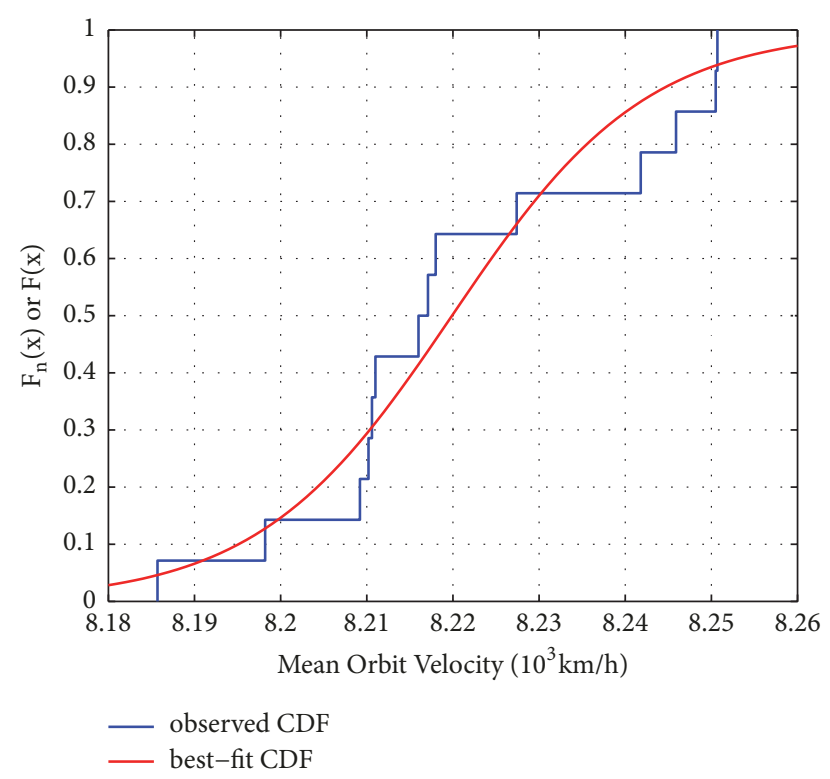

Figure 11: Comparison of the observed CDF and best-fit CDF of mean orbit velocity.

The distribution implies that average equatorial radius of the moons in Carme group is $1.67257 \mathrm{~km}$ with confidence interval [1.23204,2.1131] (we believe that the confidence interval will be smaller and shorter with continuous progress of observation technology) under the level of significance 0.05. For the true value of Erinome's equatorial radius being $1.6 \mathrm{~km}$, it is easy to find that we can use the estimated value to predict the equatorial radius or as a reference to study other relevant physical and orbital characteristics.

\section{Conclusions}

By using the one-sample K-S nonparametric test method of statistical inference, the distribution laws of the physical and orbital properties of Jupiter's moons are investigated statistically in this paper. The physical and orbital characteristics of Jupiter's moons are found to obey the Birnbaum-Saunders distribution, the logistic distribution, the Weibull distribution, and the $t$ location-scale distribution.

In addition, the probability density curves of the data distributions are also generated, and the differences in the physical and orbital characteristics of the three groups are explained in more detail.

Through a specific example, we find that some moons' missing data can be inferred by using the aforementioned distributional model and probability density function. More importantly, with the help of the distribution, it can be even helpful to predict the physical or orbital features of the undiscovered moon.

If future observations will allow for the expansion of the number of Jupiter's moons, we believe that the distribution laws will be slightly modified as potential newly discovered distribution functions fit the increased sample better, and the distributions will probably tend to be more uniform; i.e., some of the different properties follow the same distribution obviously, but these will not change dramatically over a long period of time.

\section{Appendix}

\section{A.}

See Table 7.

\section{B. Distribution Inference Results}

See Tables 8, 9, and 10

\section{Data Availability}

The data used to support the findings of this study are available from the corresponding author upon request.

\section{Conflicts of Interest}

The authors declare that they have no conflicts of interest.

\section{Acknowledgments}

The authors acknowledge the support of National Natural Science Foundation of China (NSFC) through grant Nos. 11672259, 11302187, and 11571301; the Ministry of Land and Resources Research of China in the Public Interest through grant No. 201411007; and Top-notch Academic Programs Project of Jiangsu Higher Education Institutions (TAPP) through grant No. PPZY2015B109.

\section{References}

[1] S. S. Sheppard, The Jupiter satellite and moon page, Carnegie Institution, 2017.

[2] D. B. Jupiter, "moons," NASA Planetary Science Division, 2017, https://solarsystem.nasa.gov/planets/jupiter/moons.

[3] D. Jewitt and N. Haghighipour, "Irregular satellites of the planets: Products of capture in the early solar system," Annual Review of Astronomy and Astrophysics, vol. 45, pp. 261-295, 2007.

[4] S. S. Sheppard, "Outer irregular satellites of the planets and their relationship with asteroids, comets and Kuiper Belt objects," Proceedings of the International Astronomical Union, vol. 1, no. 229, pp. 319-334, 2005.

[5] B. W. Carroll and D. A. Ostlie, An introduction to modern astrophysics, Pearson, 2nd edition, 2006.

[6] Elkins-Tanton LT, “Jupiter and Saturn,” Facts on File, 2010.

[7] D. Jewitt, S. Sheppard, and C. Porco, "Jupiter's outer satellites and Trojans," in Jupiter. The planet, satellites and magnetosphere, F. Bagenal, T. E. Dowling, and W. B. McKinnon, Eds., vol. 1, pp. 263-280, Cambridge planetary science, Cambridge, UK, 2004.

[8] Wikipedians, "Moons of Jupiter," in Jupiter, pp. 78-94, PediaPress GmbH, 2018.

[9] D. Nesvorný, J. L. A. Alvarellos, L. Dones, and H. F. Levison, "Orbital and collisional evolution of the irregular satellites," The Astronomical Journal, vol. 126, no. 1, pp. 398-429, 2003. 
[10] D. Nesvorný, C. Beaugé, and L. Dones, "Collisional origin of families of irregular satellites," The Astronomical Journal, vol. 127, no. 3, pp. 1768-1783, 2004.

[11] S. S. Sheppard and D. C. Jewitt, "An abundant population of small irregular satellites around Jupiter," Nature, vol. 423, no. 6937, pp. 261-263, 2003.

[12] B. Carry, "Density of asteroids," Planetary and Space Science, vol. 73, no. 1, pp. 98-118, 2012.

[13] P. Kankiewicz and I. Włodarczyk, "Dynamical lifetimes of asteroids in retrograde orbits," Monthly Notices of the Royal Astronomical Society, vol. 468, no. 4, pp. 4143-4150, 2017.

[14] R. V. Hogg and A. T. Craig, Introduction to mathematical statistics, Pearson Education Asia Limited and Higher Education Press, 2004.

[15] H. Xie, X. Cui, B. Wan, and J. Zhang, "Statistical analysis of radio interference of $1000 \mathrm{kV}$ UHV AC double-circuit transmission lines in foul weather," CSEE Journal of Power and Energy Systems, vol. 2, no. 2, pp. 47-55, 2016.

[16] R. R. Wilcox, "Some practical reasons for reconsidering the Kolmogorov-Smirnov test," British Journal of Mathematical and Statistical Psychology, vol. 50, no. 1, pp. 9-20, 1997.

[17] Z. W. Birnbaum and S. C. Saunders, "A new family of life distributions," Journal of Applied Probability, vol. 6, no. 2, pp. 319-327, 1969.

[18] K. B. Athreya and S. N. Lahiri, Measure Theory and Probability Theory, Springer Texts in Statistics, Springer, New York, NY, USA, 2006.

[19] List of moons in orbit around Jupiter, 2018, https://www .universeguide.com/planet/jupiter\#themoons.

[20] R. Jiang and D. N. P. Murthy, "A study of Weibull shape parameter: Properties and significance," Reliability Engineering \& System Safety, vol. 96, no. 12, pp. 1619-1626, 2011. 

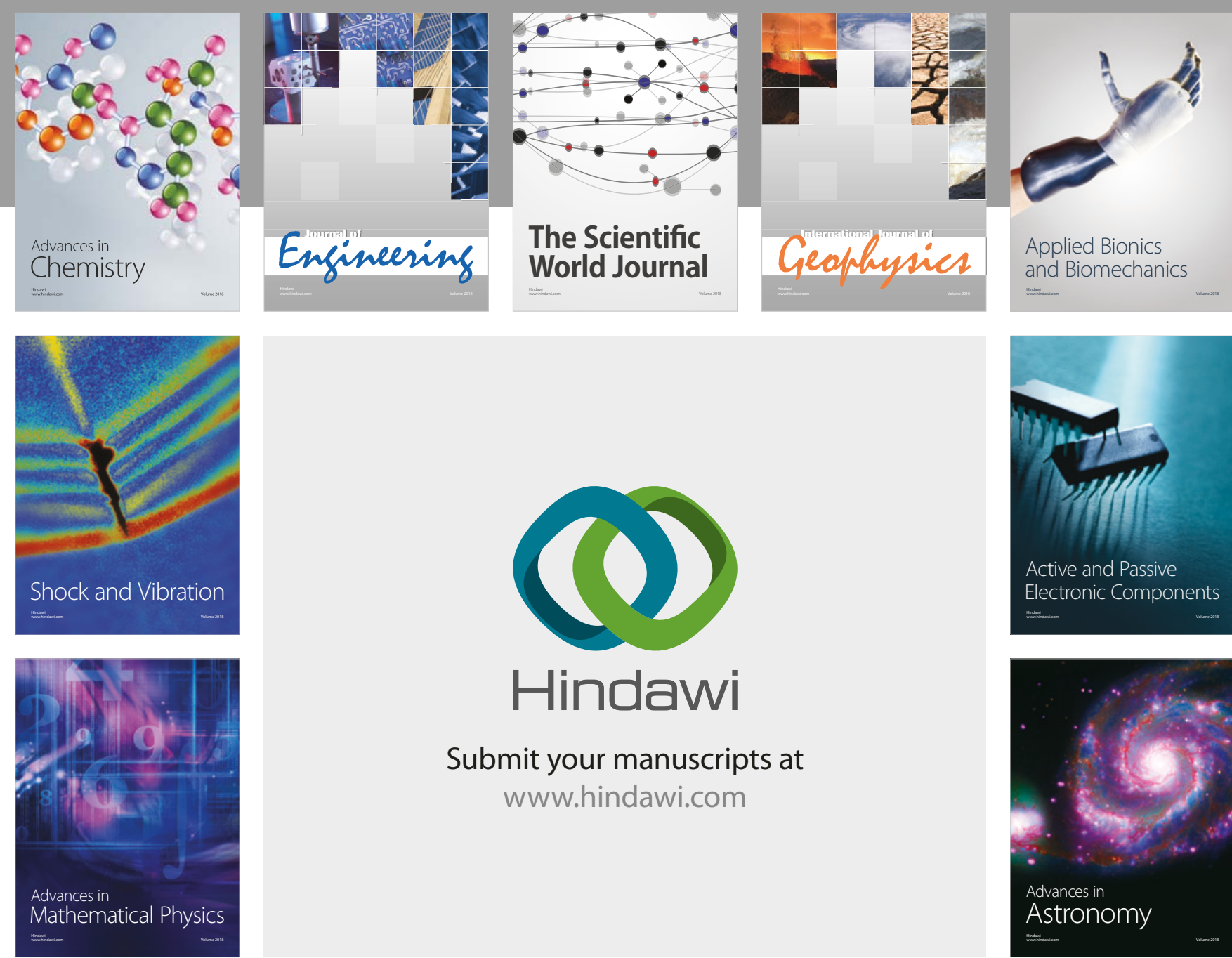

Submit your manuscripts at

www.hindawi.com

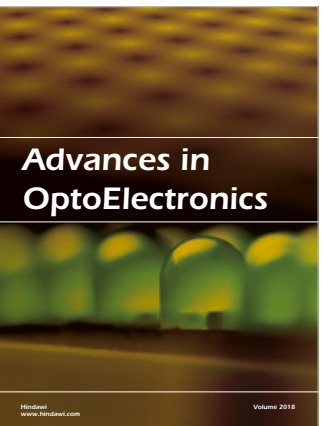

\section{Rotcting Machinery}
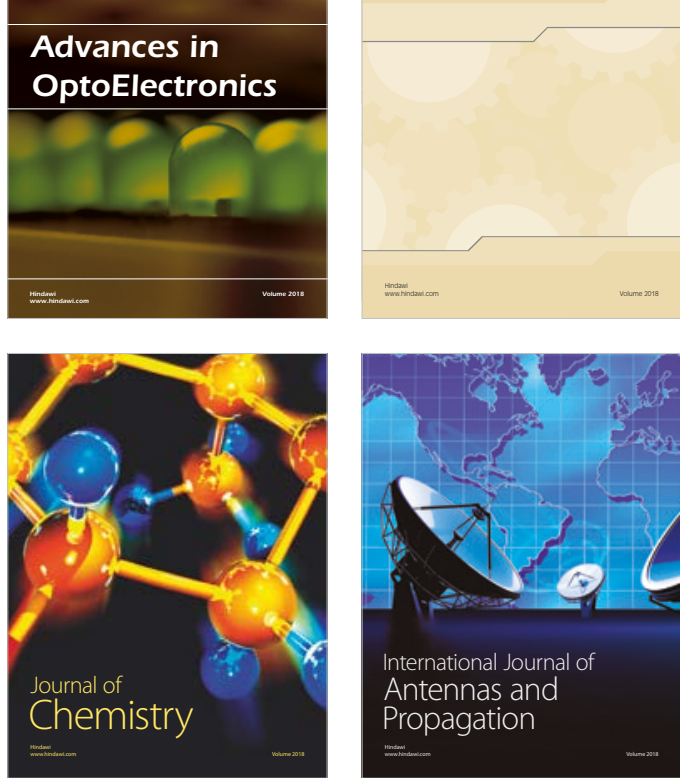

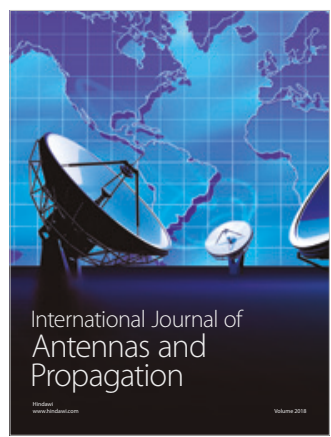

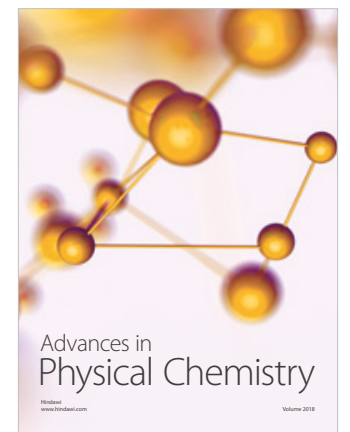

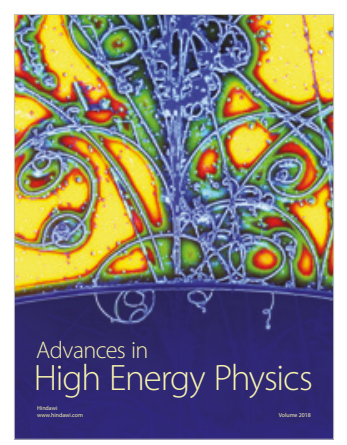

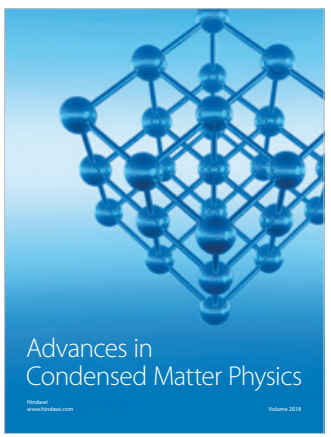

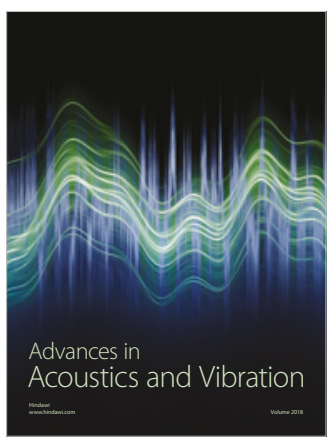

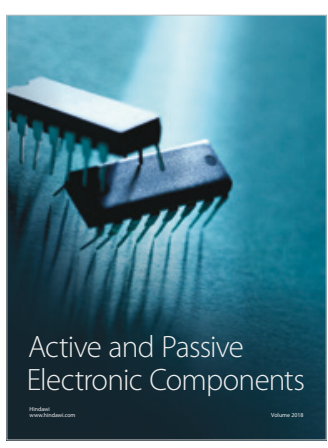
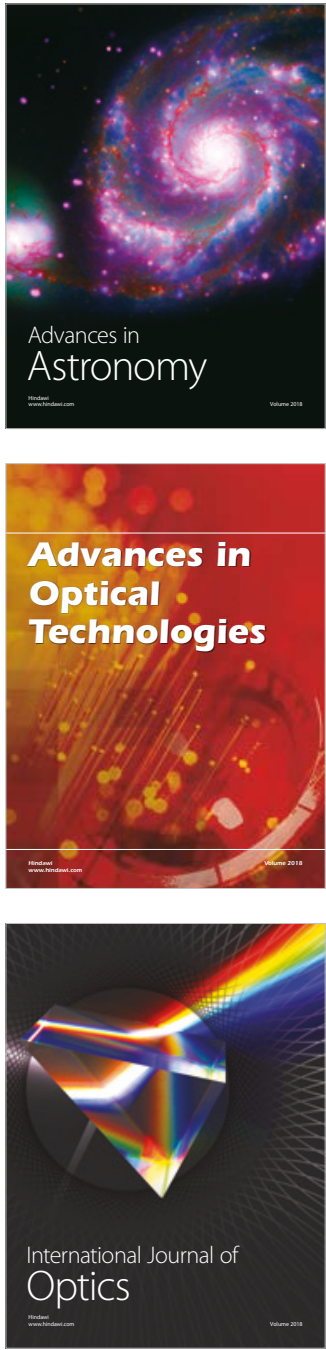